\title{
EFFECT OF SPROUTING USING SALINE WATER ON CHEMICAL COMPOSITION AND PROTEIN QUALITY AND FRACTIONATIONS OF EGYPTIAN CLOVER SPROUTS
}

\author{
El-Gebaly ${ }^{1}$, A.A.; Y.A. El-Gabry ${ }^{2}$; Sanaa A. Mahfouz ${ }^{1}$ and M.M.F. Abdallah ${ }^{3}$
}

1- Regional Center for Food and Feed, Agricultural Research Center, Giza, Egypt

2- Agronomy Dept., Fac. of Agric., Ain Shams Univ., P.O. Box 68 Hadayek Shoubra, 11241, Cairo, Egypt

3- Horticulture Dept., Fac. of Agric., Ain Shams Univ., P.O. Box 68 Hadayek Shoubra, 11241, Cairo, Egypt

Keywords: Seed sprouting, Proximate composition, Protein quality, Amino acid profile, Protein fraction

\section{ABSTRACT}

The effect of salt stress on growth proximate analysis, amino acid profile, protein quality and fractionations in 3 days etiolated clover sprout samples was investigated. Sterilized and nonsterilized clover seeds were sprouting using tap water $1000 \mathrm{ppm}$ and $2000 \mathrm{ppm} \mathrm{NaCl}$ solution. The results showed reduction effect of clover sprout characters with higher $\mathrm{NaCl}$ concentration. Clover seed sprouting increased the crude protein content using tap water or saline water for sprouting as compared with dry seeds. However using nonsterilized clover seeds for sprouting recorded the higher values of protein (44.73\%), lipids (6.21\%) and energy $(318.51 \mathrm{kcal} / \mathrm{g})$ in etiolated sprouts, while using sterilized seeds recorded higher carbohydrate (21.28\%), fiber (14.57\%) and ash (4.46\%). For amino acids, aspartic acid followed by glutamic acid were the most abundant, while Cysteine and methionine were the least in clover etiolated sprouts. Using saline water for clover seed sprouting caused increases in all amino acid compared with tap water except Methionine, aspartic acid and cysteine. For protein efficiency ratio (PER), essential amino acid index (EAAI\%) and biological value (BV), from using saline water for sprouting had the higher values than sprouts from using tap water, but the values are less than dry seeds. On the other hand nutritional index (NI) recorded the higher values in salt stress compared with both using tap water and dry clover seeds. For protein fraction in etiolated clover sprouts albumin was the major protein fraction extracted from $\mathrm{NaCl}$ 2000ppm sprout fallowed by prolamin from tap water sprouts, glutelin from $\mathrm{NaCl} 1000$ ppm sprout and globulin from tap water sprout. The clover sprout protein isolated and its fractions could have excellent applications for future product development by virtue of their nutritional and functional properties.

\section{INTRODUCTION}

As Egypt population increases rapidly and it is consider one of the main constraints for limiting land, water and food resources, it's more important to define accurately the amount and quality of protein required to meet human nutritional needs. From nutritional point of view, clover or alfalfa sprouts are considered as a high quality for the health, due to its rich nutritional profile (>50\% protein) (Cairney 1997 and Abdallah 2008).

Clover (Berrsem) was first cultivated thousands of years ago in Egypt before pyramid building (Jones et al 1982 and Abdallah (2008) reported that seed germination and production of sprouts is an old habit that was adapted 3 days of sprouting to be the optimum period for clover growing in the dark to produce etiolated sprouts. Clover sprouts are the most common usually eaten raw in salads, therefore its recommends that, prior to sprouting, seeds be disinfected by washing in solution of $2 \%$ Calcium hypochlorite $\left(\mathrm{CaCl}_{2} \mathrm{O}_{2}\right)$ (Lang et al 2000). 
In arid and semi-arid areas, salinity is the most serious a biotic stress that effects plant morphology and physiology through osmotic and ionic stress and changes biochemical response in plant (Khan et al 2013). Plants can resist osmotic stress by increasing the size of root system or reducing leaf area (Guo et al 2002; Han and Wang, 2005). Plants resist osmotic stress also by changing physiological and biochemical process such as antioxidant defense systems, solute accumulation, etc. (Hasegawa et al 2000, Hsiao and Xu, 2000 and Ibrahim et al 2017).

Proline quality evaluation aims to determine the capacity of food protein sources and diets to meet the essential amino acids needs. Compared to seeds, the sprouts have a higher quality of protein and more favorable amino acid composition (Urbano et al 2005, Wang, et al 2005; Abdallah 2009). The greatest increase during sprouting appears to be in glutamic and aspartic acids. (Rozan et al 2001; Abdallah et al 2009 and Ibrahim, 2017). The salt stress caused increase level of amino acids proline, glutamic acid, aspartic acid and arginine in cereal Simon-Sarkadi et al (2002). Moreover, during sprouting the water soluble protein content increased, but the salt soluble protein fraction decreased (Wanasundara et al 1999).

Albumins are water-soluble proteins, whereas globulins are salt $(\mathrm{NaCl})$-soluble proteins, and both were the major protein fractions, followed by storage proteins, glutelin (ar organic solution - soluble proteins) in both wheat and barley seeds (Xue Zhu et al 2006 and Kumar and Matta, 2011).

During barley seed germination Albumins and globulins degraded faster. While glutelin and prolamin fractions were not seen degradation products (Kumar and Matta, 2011).

The Present investigation were under taken for determine the changes occurring in four protein fractions during sprouting of clover seeds using saline water and chemical composition investigate and protein quality of clover sprouts. The outcomes of this study would be important as a functional food ingredients.

\section{MATERIALS AND METHODS}

This study was carried out in Horticulture Department, Faculty of Agriculture, Ain Shams University, Cairo, and the Regional center for Food and Feed (RCFF), Agricultural Research center (ARC), Giza, Egypt.in the period (2016-2017).

\section{Materials}

Egyptian clover seeds (Trifolium aelxandrium) were obtained from the Crops Research Institute Agriculture Research Center, Giza Egypt. The seeds were cleaned from all impurities for sprouting, Calcium Hypochlorite $70 \%$, Sodium chloride, absolute Ethanol and Sodium Hydroxide. Were obtained from Ahram Company, Cairo.

\section{Methods}

\section{Seed sprouting}

Sprouting of clover seeds was carried out in the glass jar as described by Abdallah (2008). The clover seeds were sterilized in Calcium Hypochlorite $2 \%$ for 20 minutes before soaking in tap water or in $\mathrm{NaCl} 1000$ and 2000 ppm solution for sprouting procedure .The other half of seeds were left unsterilized and soaking in bath tap water and $\mathrm{NaCl}$ solutions in the same sprouting procedure. Three days old Egyptian clover sprout from each Sprouting treatments and dry seeds (oven dried at $60 \mathrm{c}^{\circ}$ for $72 \mathrm{hr}$.) were ground to pass through a 40 mesh sieve, the samples were stored until analysis at $5^{\circ} \mathrm{C}$. Samples of harvested Three days old etiolated clover sprouts were taken from each treatment for measuring sprout characters (length of hypocotyl day and sprout and fresh weight of ten sprout and hypocotyl day).

\section{Proximate analysis}

Ash, total protein, lipids and crude fiber contents of samples were determined according to AOAC (2012). Total carbohydrates were determined by subtracting. Energy value was calculated using the method $[(9 \times$ fat $)+(4 \times$ carbohydrate $)+$ ( $4 \times$ protein)] as described by Chinma and Igyor (2007).

\section{Amino acid Analysis}

Amino acid determination was performed in Regional Center for Food and Feed, Agriculture Research Center, Giza, Egypt according to AOAC (2012) by using performic oxidation method (applicable to determination of amino acid (including methionine. and cystine) in feeds. Performic acaid oxidation is performed prior to hydrolysis to oxidize cystine and methionine to cysteic acid and methionine sulfone. Hydrolysis was carried out in closed conical flask for determining all amino acids other 
than tryptophan. Sample equal to $10 \mathrm{mg}$ of Protein was weighed in the conical flask and $5 \mathrm{ml}$. of per formic acid was added. The flask was closed and placed in ice bath for $16 \mathrm{~h}$. Sodium disulfite was added, $25 \mathrm{ml} \mathrm{HCL} 6 \mathrm{~N}$ was added to the oxidized mixture. The flask was placed in an oven at $110^{\circ} \mathrm{C}$ for $24 \mathrm{~h}$. The flask was then opened using Rotary evaporator to reduce the volume 5-10 $\mathrm{Ml}$ under vacuum at $60{ }^{\circ} \mathrm{C}$. Adjust the $\mathrm{PH}$ to 2.20 with sodium Hydroxide solution. Suitable volume of sodium citrate buffer ( $\mathrm{PH} 2.20$ ) was added to hydrolyzed sample. After all soluble material completely, dissolved; the sample is ready for analysis. The system used for the analysis was high performance Amino Acid analyzer (Biochrom 30).

\section{Nutritional quality of clover sprouts}

Sprouts samples Nutritional quality was determined using amino acid profiles and calculated essential amino acid index (EAAI) according to Labuda et al (1982) method according to the following equation:

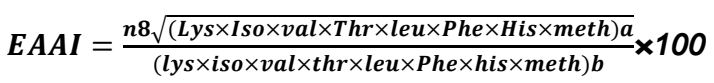

Where:

$\mathrm{n}=$ number of essential amino acids, $a, \ldots=$ represent the concentration of essential amino acids[lysine, isoleucine, valine, threonine, leucine, phenylalanine, histidine and methionine]in test sample and $, b, \ldots=$ content of the same amino acids in standard protein (\%) (Tahany, Aly. et al 2018).

Biological value was calculated according to Oser (1959) using the following equation:

BV $=1.09 \times$ Essential amino acid index (EAAI) 11.7

The nutritional index percentage of sprout samples was determined using the following equation according to ljarorimi and Keshinro., (2013).

(NI)Nutritional index $(\%)=\frac{\text { EAAI } \% \text { protein }}{100}$

Protein Efficiency Ratio (C-PER) was estimated according to the regression equations developed by Alsmeyer et al (1974) as given below:
$P E R=-0.468+0.454($ LEU $)-0.105(T Y R)$

\section{Calculation of other protein quality parameters}

From the results obtained for amino acids profile in seed sprouts. The total amino acids (TAA); total essential amino acids (TEAA); total neutral amino acids (TNAA); total acidic amino acids (TAAA); total aromatic amino acids (TArAA); and total basic amino acids (TBAA); were estimated. The ratio of (TEAA/TAA) and (\% Cysteine/TSAA), also estimated.

Protein fractionation: using the procedure of Osborne (1909) with minor modifications was determined according to Tounkara et al (2013).

\section{Statistical analysis}

The data were statistically analyzed by analysis of variance using completely randomized design and least significant difference (L.S.D) at 0.05 levels according to the method described by Snedecor and Cochran (1980).

\section{RESULTS AND DISCUSSION}

\section{Effect of sprouting using saline water on sprout characters}

Data in Table (1) showed no significant difference $(p \leq 0.05)$ between sterilized and non-sterilized seed on 3days old clover etiolated sprout.

The length Sprout $(6.12 \mathrm{~cm})$ and hypocotyl length $(3.10 \mathrm{~cm})$ and heavier fresh weight $(281.9$ and $237.8 \mathrm{mg}$ respectively) were observed when using tap water for sprouting as compared with using 1000 and 2000 ppm NaCl. The interaction effect between seed sterilization and $\mathrm{NaCl}$ concentrations showed the higher sprout length characters in sterilization with using tap water but in sprouts fresh weight the non-sterilized seeds with tap water recorded the heavier weight. The lowest sprout characters was recorded with increasing $\mathrm{NaCl}$ concentration (2000 ppm).Similar results on the reduction effect of sprout characters with higher $\mathrm{NaCl}$ concentration were reported by Ghoulam and Fares (2001); Ibrahim (2017) and Abd Elghany (2017). 
Table 1. Effect of $\mathrm{NaCl}$ concentration in sprouting solution clover etiolated sprout sterilized seeds characters (average of two experiments)

\begin{tabular}{|c|c|c|c|c|c|}
\hline $\begin{array}{l}\text { Sterilization } \\
\text { (ste) }\end{array}$ & $\begin{array}{c}\mathrm{NaCl} \\
\text { concentration }\end{array}$ & $\begin{array}{l}\text { Hypocotyl } \\
\text { length } \\
\text { (cm) }\end{array}$ & $\begin{array}{c}\text { Sprout } \\
\text { length } \\
\text { (cm) }\end{array}$ & $\begin{array}{c}10 \text { Hypocotyl } \\
\text { fresh weight } \\
(\mathrm{mg})\end{array}$ & $\begin{array}{l}10 \text { Sprout } \\
\text { fresh weight } \\
(\mathrm{mg})\end{array}$ \\
\hline $\begin{array}{l}\text { Sterilized } \\
\text { seeds }\end{array}$ & Tap water & $3.23^{\mathrm{a}}$ & $6.27^{\mathrm{a}}$ & $237.18^{\mathrm{a}}$ & $279.00^{a}$ \\
\hline \multirow[t]{2}{*}{$\mathrm{NaCl}, \mathrm{ppm}$} & 1000 & $2.5^{\mathrm{c}}$ & $5.10^{\mathrm{e}}$ & $198.16^{b}$ & $237.66^{\mathrm{bc}}$ \\
\hline & 2000 & $2.47^{\mathrm{c}}$ & $5.23^{\mathrm{de}}$ & $199.93^{b}$ & $235.69^{c}$ \\
\hline Mean & & $2.73^{\mathrm{A}}$ & $5.53^{\mathrm{A}}$ & $211.76^{\mathrm{A}}$ & $250.78^{A}$ \\
\hline $\begin{array}{c}\text { Non- } \\
\text { sterilized } \\
\text { seeds }\end{array}$ & Tap water & $2.97^{\mathrm{ab}}$ & $5.97^{\mathrm{ab}}$ & $238.40^{\mathrm{a}}$ & $284.77^{\mathrm{a}}$ \\
\hline \multirow[t]{2}{*}{$\mathrm{NaCl}, \mathrm{ppm}$} & 1000 & $2.77^{\mathrm{bc}}$ & $5.61^{b c}$ & $222.55^{\mathrm{a}}$ & $256.33^{\mathrm{b}}$ \\
\hline & 2000 & $2.6^{\mathrm{c}}$ & $5.51^{\mathrm{cd}}$ & $194.03^{b}$ & $230.95^{\mathrm{C}}$ \\
\hline Mean & & $2.78^{\mathrm{A}}$ & $5.70^{\mathrm{A}}$ & $218.33^{A}$ & $257.35^{\mathrm{A}}$ \\
\hline \multirow{3}{*}{$\begin{array}{l}\text { Average } \\
\mathrm{NaCl}, \mathrm{ppm}\end{array}$} & Tap water & $3.1^{\mathrm{A}}$ & $6.12^{\mathrm{A}}$ & $237.79^{\mathrm{A}}$ & $281.89^{A}$ \\
\hline & 1000 & $2.63^{\mathrm{B}}$ & $5.36^{\mathrm{B}}$ & $210.35^{\mathrm{B}}$ & $247.00^{\mathrm{B}}$ \\
\hline & 2000 & $2.53^{\mathrm{B}}$ & $5.37^{\mathrm{B}}$ & $196.98^{\mathrm{B}}$ & $233.32^{C}$ \\
\hline \multirow{3}{*}{ L.S.D (0.05) } & Ste & NS & NS & NS & NS \\
\hline & $\mathrm{NaCl}$ & 0.23 & 0.27 & 13.77 & 13.65 \\
\hline & ste $\times \mathrm{NaCl}$ & 0.33 & 0.38 & 19.47 & 19.30 \\
\hline
\end{tabular}

Means in each column followed by the same letter are not significantly different at the $5 \%$ level $(p \leq 0.05)$

NS= non -significant.

\section{Proximate analysis of sprout}

The results of the proximate analysis of clover etiolated sprouts from sterilized and non-sterilized seeds and dry seeds are summarized in Table (2). Non-sterilized seeds recorded the higher values of moisture content $(9.48 \%)$, crude protein $(44.73 \%)$, lipids $(6.21 \%)$ and energy $(318.51 \mathrm{kcal} / \mathrm{g})$, while sterilized seeds recorded higher carbohydrate $(21.28 \%)$, fiber $(14.57 \%)$, ash $(4.46 \%)$. Using saline water (1000 and $2000 \mathrm{ppm} \mathrm{NaCl}$ ) for sprouting decreased sprout moisture percent and data was more pronounced using sterilized seeds. On the other hand, using tap water or saline water increased crude protein value as compared with dry seed, with more pronounced using tap water for sprouting which recorded more than $24 \%$ increment in protein compared with dry seeds. The highest values of crude protein in 3days old clover etiolated sprouts, could be due to a decrease in dry matter through respiration of young sprouts, due to there were no nitrogen source added externally to the water and saline solution used for irrigation during sprouting .This crude protein \% increase was therefore not a likely true increase (Chavan and Kadam 1989, Abdallah 2008 and Dung et al 2010). Carbohydrate values by difference recorded opposite results with protein and showed clear eased volute in sprouts compared with dry seeds speedily when using tap water for sprouting (Table 2). Lipid, fiber and ash value increased in clover sprouts than dry seeds. The fiber increased with increasing $\mathrm{NaCl}$ concentration, while ash decreased with increasing $\mathrm{NaCl}$ concentration. Clover etiolated sprouts relabeled lower energy value as compared with dry seeds. These observation may be clue to decreased in carbohydrate content. 
Protein Quality and Fractionations of Egyptian Clover Sprouts

Table 2. Proximate analysis ( $\mathrm{g} / 100 \mathrm{~g}$ ), and Energy $(\mathrm{Kcal} / \mathrm{g})$ of 3 day old etiolated clover sprout

\begin{tabular}{|c|c|c|c|c|c|c|c|c|}
\hline Sterilize (ste) & $\begin{array}{c}\mathrm{NaCl} \\
\text { concentra }\end{array}$ & Moisture & $\begin{array}{c}\text { Crude } \\
\text { protein }\end{array}$ & Carbohy & Lipids & Fiber & Ash & $\begin{array}{r}\text { Energy } \\
\text { (Kcal/g) }\end{array}$ \\
\hline $\begin{array}{c}\text { Sterilized seed } \\
\text { NaCl, ppm } \\
\text { Mean }\end{array}$ & $\begin{array}{c}\text { Dry seed } \\
\text { Tap water } \\
1000 \\
2000\end{array}$ & $\begin{array}{c}9.58^{\mathrm{b}} \\
9.23^{\mathrm{cd}} \\
8.93^{\mathrm{f}} \\
8.98^{\mathrm{ef}} \\
9.18^{\mathrm{B}} \\
\end{array}$ & $\begin{array}{l}38.57^{f} \\
47.45^{b} \\
47.57^{b} \\
44.36^{e} \\
44.49^{B} \\
\end{array}$ & $\begin{array}{l}33.12^{\mathrm{a}} \\
16.16^{\mathrm{e}} \\
17.37^{\mathrm{d}} \\
18.46^{\mathrm{c}} \\
21.28^{\mathrm{A}} \\
\end{array}$ & $\begin{array}{l}4.28^{\mathrm{g}} \\
6.77^{\mathrm{d}} \\
6.26^{\mathrm{e}} \\
6.80^{\mathrm{C}} \\
6.03^{\mathrm{B}} \\
\end{array}$ & $\begin{array}{c}10.59^{\mathrm{g}} \\
14.84^{\mathrm{f}} \\
15.88^{\mathrm{b}} \\
16.95^{\mathrm{a}} \\
14.57^{\mathrm{A}} \\
\end{array}$ & $\begin{array}{l}3.85^{\mathrm{f}} \\
5.55^{\mathrm{a}} \\
3.99^{\mathrm{e}} \\
4.45^{\mathrm{d}} \\
4.46^{\mathrm{A}} \\
\end{array}$ & $\begin{array}{c}325.34^{a} \\
315.34^{\text {cd }} \\
316.09^{c} \\
312.47^{f} \\
317.31^{B} \\
\end{array}$ \\
\hline $\begin{array}{c}\text { Non- } \\
\text { Sterilized } \\
\text { Seed } \\
\mathrm{NaCl}, \text { ppm } \\
\text { Mean } \\
\end{array}$ & $\begin{array}{c}\text { Dry seed } \\
\text { Tap water } \\
1000 \\
2000\end{array}$ & $\begin{array}{l}9.58^{\mathrm{b}} \\
9.95^{\mathrm{a}} \\
9.10^{\mathrm{de}} \\
9.28^{\mathrm{C}} \\
9.48^{\mathrm{A}}\end{array}$ & $\begin{array}{l}38.57^{f} \\
48.28^{a} \\
46.48^{\mathrm{C}} \\
45.57^{\mathrm{d}} \\
44.73^{\mathrm{A}}\end{array}$ & $\begin{array}{l}33.12^{\mathrm{a}} \\
15.07^{\mathrm{f}} \\
16.29^{\mathrm{e}} \\
19.23^{\mathrm{b}} \\
20.93^{\mathrm{B}}\end{array}$ & $\begin{array}{l}4.28^{\mathrm{g}} \\
6.84^{\mathrm{b}} \\
7.56^{\mathrm{a}} \\
6.15^{\mathrm{f}} \\
6.21^{\mathrm{A}}\end{array}$ & $\begin{array}{l}10.59^{\mathrm{g}} \\
15.10^{\mathrm{e}} \\
15.51^{\mathrm{d}} \\
15.78^{\mathrm{C}} \\
14.25^{\mathrm{B}}\end{array}$ & $\begin{array}{l}3.85^{f} \\
4.75^{\mathrm{c}} \\
5.05^{\mathrm{b}} \\
3.99^{\mathrm{e}} \\
4.41^{\mathrm{B}}\end{array}$ & $\begin{array}{l}325.34^{\mathrm{a}} \\
315.00^{\mathrm{de}} \\
319.15^{\mathrm{b}} \\
314.54^{\mathrm{e}} \\
318.51^{\mathrm{A}}\end{array}$ \\
\hline $\begin{array}{c}\text { Average } \\
\mathrm{NaCl}, \mathrm{ppm}\end{array}$ & $\begin{array}{c}\text { Dry seed } \\
\text { Tap water } \\
1000 \\
2000\end{array}$ & $\begin{array}{l}9.58^{\mathrm{B}} \\
9.59^{\mathrm{A}} \\
9.02^{\mathrm{C}} \\
9.13^{\mathrm{B}}\end{array}$ & $\begin{array}{l}38.57^{F} \\
47.87^{A} \\
47.03^{B} \\
44.96^{C}\end{array}$ & $\begin{array}{l}33.12^{A} \\
15.62^{B} \\
16.83^{C} \\
18.85^{B}\end{array}$ & $\begin{array}{l}4.28^{\mathrm{G}} \\
6.81^{\mathrm{B}} \\
6.91^{\mathrm{A}} \\
6.48^{\mathrm{C}}\end{array}$ & $\begin{array}{l}10.59^{\mathrm{G}} \\
14.97^{\mathrm{C}} \\
15.70^{\mathrm{B}} \\
16.37^{\mathrm{A}}\end{array}$ & $\begin{array}{l}3.85^{\mathrm{F}} \\
5.15^{\mathrm{A}} \\
4.52^{\mathrm{B}} \\
4.22^{\mathrm{C}}\end{array}$ & $\begin{array}{l}325.34^{A} \\
315.17^{C} \\
317.62^{B} \\
313.50^{D}\end{array}$ \\
\hline LSD (0.05) & $\begin{array}{c}\text { Ste } \\
\mathrm{NaCl} \\
\text { Ste } \times \mathrm{NaCl}\end{array}$ & $\begin{array}{l}0.081 \\
0.114 \\
0.161\end{array}$ & $\begin{array}{l}0.077 \\
0.108 \\
0.153\end{array}$ & $\begin{array}{l}0.135 \\
0.191 \\
0.269\end{array}$ & $\begin{array}{l}0.011 \\
0.015 \\
0.021\end{array}$ & $\begin{array}{l}0.026 \\
0.037 \\
0.053\end{array}$ & $\begin{array}{l}0.038 \\
0.053 \\
0.075\end{array}$ & $\begin{array}{l}0.389 \\
0.550 \\
0.778\end{array}$ \\
\hline
\end{tabular}

Means in each column followed by the same letter are not significantly different at the $5 \%$ level $(p \leq 0.05)$.

\section{Amino acid profile and quality of sprout}

The amino acid profile of 3 days etiolated Egyptian clovers sprout samples are presented in Table (3). Data showed that sterilized clovers seeds before sprouting decreased four amino acids (Leucine, Glutamic acid, Argenine, and Glycine) and increased aspartic acid only as compared with non-sterilized seeds. Other amino acids not affected by seed sterilized (Table 3).

Concerning the effect of $\mathrm{NaCl}$ concentration effects data showed that sprouting in tap water and $\mathrm{NaCl}$ especially in low concentration (1000 ppm) decreased all essential amino acid content and non-essential amino acid compared with dry seeds, except high $\mathrm{NaCl}$ concentration (2000ppm) for Valine, Alanine, Serine and Tyrosine amino acids.. However, opposite results recorded for aspartic acid which recorded the lowest value in dry seeds and highest value in $\mathrm{NaCl} 2000$ ppm.

The interaction between seed sterilization and $\mathrm{NaCl}$ concentration showed that non sterilized seeds sprouting using tap water recorded the lowest essential and non-essential amino acid value expect Methionine, Aspartic acid, and Cysteine amino acids which recorded the lowest values with using $\mathrm{NaCl}$ at higher concentration (2000 ppm) Leucine and using lower $\mathrm{NaCl}$ concentration (1000 ppm) for Cysteine and in dry seeds for Aspartic acid. On the other hand dry seed sample recorded the higher essential amino acids value except Valine and Phenylalanine which showed the high value with higher $\mathrm{NaCl}$ concentration (2000 ppm) interact with non-sterilized seeds. Also, dry seed sample recorded the higher non-essential amino acids value, except aspartic, alanine, serine and tyrosine which recorded the higher values with higher $\mathrm{NaCl}$ concentration (2000 ppm) interact with non-sterilized seeds, Also for Alanine, Serine and Tyrosine, but the higher values for aspartic acid was recorded with lower $\mathrm{NaCl}$ concentration (1000 ppm) interact with sterilized seeds (Table 3).

Using saline water at higher concentration (2000 ppm $\mathrm{NaCl}$ ) for non-sterilized seed sprouting increased the content of Valine, Phenylalanine, Alanine, Serine and Tyrosine pile using tap water for sprouting decreased of almost all amino acids except Methionine, Aspartic acid and Cysteine.

Data in Table (3) also showed that aspartic acid was found to be the most abundant in sterilized or non-sterilized seed sprouts followed by glutamic acid. The higher aspartic and glutamic acid was recorded with higher $\mathrm{NaCl}$ concentration sprouts (13.77 and $10.82 \mathrm{~g} / 100 \mathrm{~g}$, respectively). 
Opposite results was shown in dry seeds which recorded lower Aspartic acid $(9.05 \mathrm{~g} / 100 \mathrm{~g})$ compared with Glutamic acid $(14.47 \mathrm{~g} / 100 \mathrm{~g})$.

An observation in Aspartic acid /Glutamic acid showed that the level of Aspartic appeared to affect the glutamic (Table 3). The lowest Aspartic acid / Glutamic acid was recorded in dry seeds (0.62) followed by sprouts using $\mathrm{NaCl}$ during sprouting (1.27), while, the highest Aspartic acid / Glutamic acid was recorded in clover sprouts using tap water for sprouting.

Table 3. Amino acids composition ( $\mathrm{g} / 100 \mathrm{~g}$ protein) of 3 days etiolated Egyptian clover sprouts

\begin{tabular}{|c|c|c|c|c|c|c|c|c|c|c|}
\hline Para met & EAA & Ile & Ther & Val & Phe & Lys & Leu & Meth & His & TEAA \\
\hline \multirow[t]{2}{*}{$\begin{array}{l}\text { Sterilized } \\
\text { Seeds }\end{array}$} & Dry seed & $3.26^{a}$ & $2.57^{a}$ & $3.91^{b}$ & $3.94^{\mathrm{a}}$ & $5.53^{\mathrm{a}}$ & $5.75^{\mathrm{a}}$ & $0.98^{a}$ & $2.56^{a}$ & $28.53^{a}$ \\
\hline & Tap water & $2.87^{\mathrm{c}}$ & $2.19^{d}$ & $3.65^{d}$ & $3.71^{\mathrm{b}}$ & $3.77^{\mathrm{e}}$ & $4.85^{\mathrm{cd}}$ & $0.78^{b}$ & $2.17^{d}$ & $24.00^{c}$ \\
\hline \multirow[t]{2}{*}{$\mathrm{NaCl}, \mathrm{ppm}$} & 1000 & $2.75^{d}$ & $2.26^{\mathrm{cd}}$ & $3.68^{\mathrm{cd}}$ & $3.59^{c}$ & $3.95^{\mathrm{cd}}$ & $4.61^{\mathrm{e}}$ & $0.91^{a b}$ & $2.25^{\mathrm{c}}$ & $24.01^{c}$ \\
\hline & 2000 & $2.86^{\mathrm{c}}$ & $2.23^{\mathrm{cd}}$ & $3.77^{c}$ & $3.63^{c}$ & $3.88^{\mathrm{de}}$ & $4.78^{d}$ & $0.81^{b}$ & $2.13^{\mathrm{de}}$ & $24.11^{c}$ \\
\hline Mean & & $2.93^{\mathrm{A}}$ & $2.31^{\mathrm{A}}$ & $3.75^{\mathrm{A}}$ & $3.71^{\mathrm{A}}$ & $4.28^{\mathrm{A}}$ & $5.00^{\mathrm{B}}$ & $0.87^{\mathrm{A}}$ & $2.27^{\mathrm{A}}$ & $28.53^{\mathrm{A}}$ \\
\hline \multirow{4}{*}{$\begin{array}{c}\text { Non } \\
\text { Sterilized } \\
\text { Seeds } \\
\mathrm{NaCl}, \mathrm{ppm}\end{array}$} & Dry s & $3.26^{a}$ & $2.57^{a}$ & $3.91^{b}$ & $3.94^{\mathrm{a}}$ & $5.53^{\mathrm{a}}$ & $5.75^{\mathrm{a}}$ & $0.98^{\mathrm{a}}$ & $2.56^{\mathrm{a}}$ & $28.53^{\mathrm{a}}$ \\
\hline & Tap water & $2.59^{\mathrm{e}}$ & $2.03^{\mathrm{e}}$ & $3.43^{\mathrm{e}}$ & $3.42^{d}$ & $3.42^{f}$ & $4.47^{f}$ & $0.89^{\mathrm{ab}}$ & $2.03^{f}$ & $22.29^{d}$ \\
\hline & 1000 & $2.86^{\mathrm{c}}$ & $2.30^{c}$ & $3.70^{\text {cd }}$ & $3.65^{b c}$ & $4.06^{c}$ & $4.95^{\mathrm{c}}$ & $0.82^{b}$ & $2.07^{\text {ef }}$ & $24.55^{c}$ \\
\hline & 2000 & $3.14^{b}$ & $2.43^{\mathrm{b}}$ & $4.15^{\mathrm{a}}$ & $3.97^{\mathrm{a}}$ & $4.36^{b}$ & $5.29^{b}$ & $0.83^{b}$ & $2.46^{\mathrm{b}}$ & $26.77^{b}$ \\
\hline Mean & & $2.96^{\mathrm{A}}$ & $2.33^{A}$ & $3.79^{\mathrm{A}}$ & $3.74^{\mathrm{A}}$ & $4.34^{\mathrm{A}}$ & $5.12^{\mathrm{A}}$ & $0.88^{A}$ & $2.28^{A}$ & $25.53^{\mathrm{A}}$ \\
\hline \multirow{4}{*}{$\begin{array}{c}\text { Average } \\
\mathrm{NaCl}, \mathrm{ppm}\end{array}$} & Dry seed & $3.26^{\mathrm{A}}$ & $2.57^{\mathrm{A}}$ & $3.91^{B}$ & $3.94^{\mathrm{A}}$ & $5.53^{A}$ & $5.75^{A}$ & $0.98^{A}$ & $2.56^{A}$ & $28.53^{A}$ \\
\hline & Tap water & $2.73^{\mathrm{D}}$ & $2.11^{\mathrm{C}}$ & $3.54^{\mathrm{C}}$ & $3.56^{\mathrm{D}}$ & $3.60^{\mathrm{D}}$ & $4.66^{\mathrm{D}}$ & $0.83^{\mathrm{B}}$ & $2.10^{\mathrm{D}}$ & $23.14^{\mathrm{D}}$ \\
\hline & 1000 & $2.81^{C}$ & $2.28^{\mathrm{B}}$ & $3.69^{B}$ & $3.62^{C}$ & $4.00^{C}$ & $4.78^{C}$ & $0.86^{\mathrm{B}}$ & $2.16^{C}$ & $24.28^{C}$ \\
\hline & 2000 & $3.00^{\mathrm{B}}$ & $2.33^{\mathrm{B}}$ & $3.96^{\mathrm{A}}$ & $3.80^{\mathrm{B}}$ & $4.12^{\mathrm{B}}$ & $5.04^{B}$ & $0.82^{\mathrm{B}}$ & $2.29^{B}$ & $25.44^{B}$ \\
\hline \multirow[t]{3}{*}{$\operatorname{LSD}(0.05)$} & St & NS & NS & NS & NS & NS & 0.06 & NS & NS & NS \\
\hline & $\mathrm{NaCl}$ & 0.07 & 0.06 & 0.07 & 0.05 & 0.1 & 0.08 & 0.1 & 0.04 & 0.47 \\
\hline & Ste $X \mathrm{NaCl}$ & 0.1 & 0.08 & 0.1 & 0.07 & 0.15 & 0.12 & 0.15 & 0.06 & 0.67 \\
\hline
\end{tabular}

Means in each column followed by the same letter are not significantly different at the $5 \%$ level $(p \leq 0.05)$. Ile= Isoleucine, Ther= Therionine, Val= Valine, Phe= Phenylalanine, Lys $=$ Lysine, Leu= Leucine, Meth $=$ Methionine, His= Histidine, TEAA = Total Essential Amino Acids and NS= non -significant.

Cont. Table .3.

\begin{tabular}{|c|c|c|c|c|c|c|c|c|c|c|c|c|}
\hline Par & NEAA & sp & Ala & Glu & Ser & Arg & Pro & Gly & Cys & Tyr & EAA & TAA \\
\hline \multirow{4}{*}{$\begin{array}{l}\text { Sterilized } \\
\text { Seeds } \\
\mathrm{NaCl}, \mathrm{ppm}\end{array}$} & Dry seed & $9.05^{\mathrm{e}}$ & $3.59^{\mathrm{ab}}$ & $4.47^{a}$ & $3.47^{b}$ & $7.92^{\mathrm{a}}$ & $3.66^{a}$ & $3.58^{a}$ & $1.63^{\mathrm{a}}$ & $3.19^{\mathrm{ab}}$ & $50.55^{\mathrm{a}}$ & $79.05^{a}$ \\
\hline & & $14.02^{b}$ & $3.46^{c}$ & $10.11^{\mathrm{c}}$ & $3.33^{b c}$ & $4.99^{\mathrm{ef}}$ & $2.68^{b c}$ & $2.87^{\mathrm{c}}$ & $1.41^{\mathrm{b}}$ & $3.01^{c}$ & $45.26^{c}$ & $69.27^{\mathrm{c}}$ \\
\hline & 1000 & $14.43^{\mathrm{a}}$ & $3.45^{\mathrm{c}}$ & $9.53^{\mathrm{cd}}$ & $3.46^{b}$ & $5.15^{\mathrm{de}}$ & $2.75^{b c}$ & $2.80^{c}$ & $0.45^{d}$ & $3.07^{b c}$ & $45.19^{c}$ & $09 . \angle 0$ \\
\hline & 2000 & $13.89^{b}$ & $3.57^{\mathrm{ab}}$ & $9.88^{c}$ & $3.18^{c}$ & $5.23^{d}$ & $2.61^{c}$ & $2.86^{c}$ & $0.57^{d}$ & $3.04^{c}$ & $45.24^{c}$ & $69.35^{c}$ \\
\hline Mean & & 12.8 & $3.52^{\mathrm{A}}$ & $10.99^{B}$ & $3.36^{A}$ & $5.82^{\mathrm{B}}$ & $2.92^{\mathrm{A}}$ & $3.03^{B}$ & $1.01^{\mathrm{A}}$ & $3.08^{A}$ & $46.57^{\mathrm{A}}$ & $71.73^{A}$ \\
\hline \multirow{4}{*}{$\begin{array}{c}\text { Non } \\
\text { Sterilized } \\
\text { seeds } \\
\mathrm{NaCl}, \mathrm{ppm}\end{array}$} & & $9.05^{\mathrm{e}}$ & $3.59^{\mathrm{ab}}$ & $14.47^{a}$ & $3.47^{b}$ & $7.92^{a}$ & $3.66^{a}$ & $3.58^{a}$ & $1.63^{\mathrm{a}}$ & $3.19^{\mathrm{ab}}$ & $50.55^{\mathrm{a}}$ & $79.05^{a}$ \\
\hline & $\mathbf{T}$ & $13.37^{c}$ & $3.19^{d}$ & $9.07^{d}$ & $2.77^{d}$ & $4.78^{f}$ & $2.37^{d}$ & $2.74^{c}$ & $0.78^{\mathrm{c}}$ & $2.82^{d}$ & $42.55^{d}$ & $64.84^{d}$ \\
\hline & 1000 & $12.12^{d}$ & $3.48^{b c}$ & $11.24^{b}$ & $3.48^{b}$ & $5.51^{\mathrm{c}}$ & $2.81^{b}$ & $3.49^{a}$ & $0.56^{d}$ & $2.99^{c}$ & $45.60^{c}$ & $70.15^{\mathrm{C}}$ \\
\hline & & $13.66^{b c}$ & $3.61^{a}$ & $11.76^{b}$ & $3.85^{\mathrm{a}}$ & $5.86^{b}$ & $2.67^{b c}$ & $3.14^{\mathrm{b}}$ & $0.96^{c}$ & $3.22^{\mathrm{a}}$ & $48.35^{\mathrm{b}}$ & $75.13^{b}$ \\
\hline Mean & & $12.05^{\mathrm{B}}$ & $3.47^{\mathrm{A}}$ & $11.63^{\mathrm{A}}$ & $3.39^{A}$ & $6.02^{\mathrm{A}}$ & $2.88^{A}$ & $3.24^{\mathrm{A}}$ & $0.98^{\mathrm{A}}$ & $3.05^{A}$ & $46.77^{\mathrm{A}}$ & $72.31^{A}$ \\
\hline \multirow{4}{*}{$\begin{array}{c}\text { Average } \\
\mathrm{NaCl}, \mathrm{ppm}\end{array}$} & ry & $9.05^{\mathrm{E}}$ & $3.59^{\mathrm{AB}}$ & $14.47^{\mathrm{A}}$ & $3.47^{B}$ & $7.92^{\mathrm{A}}$ & $3.66^{A}$ & $3.58^{\mathrm{A}}$ & $1.63^{\mathrm{A}}$ & $3.19^{\mathrm{AB}}$ & $50.55^{\mathrm{A}}$ & $79.05^{A}$ \\
\hline & & $13.69^{A}$ & $3.32^{C}$ & $9.59^{C}$ & $3.05^{B}$ & $4.88^{\mathrm{D}}$ & $2.52^{\mathrm{C}}$ & $2.80^{\mathrm{D}}$ & $1.09^{B}$ & $2.91^{\mathrm{C}}$ & $43.90^{\mathrm{D}}$ & $67.05^{\mathrm{D}}$ \\
\hline & & $13.23^{\mathrm{B}}$ & $3.47^{\mathrm{B}}$ & $10.38^{B}$ & $3.47^{\mathrm{A}}$ & $5.33^{C}$ & $2.78^{\mathrm{B}}$ & $3.15^{\mathrm{B}}$ & $0.50^{\mathrm{D}}$ & $3.03^{B}$ & $45.39^{C}$ & $69.68^{C}$ \\
\hline & & $13.77^{\mathrm{A}}$ & $3.59 \mathrm{~A}$ & $10.82^{\mathrm{B}}$ & $3.52^{\mathrm{A}}$ & $5.54^{\mathrm{B}}$ & $2.64^{C}$ & $3.00^{C}$ & $0.76^{\mathrm{C}}$ & $3.13^{\mathrm{A}}$ & $46.80^{\mathrm{B}}$ & $72.24^{B}$ \\
\hline \multirow{3}{*}{ LSD (0.05) } & & 0.1 & NS & 0.35 & NS & 0.1 & NS & 0.08 & NS & NS & $\mathrm{NS}$ & NS \\
\hline & $\mathrm{NaCl}$ & 0.25 & 0.07 & 0,5 & 0.13 & 0.16 & 0.11 & 0.11 & 0.13 & 0.09 & 0.86 & 1.3 \\
\hline & & 6 & 0.11 & 0.71 & 0.19 & 0.22 & 0.16 & 0.16 & 0.1 & 0.13 & 1.22 & 1.84 \\
\hline
\end{tabular}

Means in each column followed by the same letter are not significantly different at the $5 \%$ level ( $p \leq 0.05)$. Asp= aspartic acid, Ala= Alanine, Glu= glutamic acid, Ser= serine, Arg= argenine, Pro= proline, Gly= glycine, Cys= cysteine, Tyr= tyrosine, NEAA= non-essential amino, TAA= total amino acid and NS=non-significant. 
The results of amino acid quality of clover seeds and etiolated sprouts are presented in Table (4). Dry clover seeds and sprouts using higher saline solution concentration (2000 ppm $\mathrm{NaCl}$ ) showed the highest percentage ratio of essential amino acid to the total amino acid (TEAA/TAA) about $36.1 \%$ and $35.2 \%$, respectively. These values were above the $26 \%$ for ideal protein for children and $11 \%$ for adult (FAO/WHO/UNU, 1985). However dry seeds and higher $\mathrm{NaCl}$ concentration sprouts recorded lower TNEAA/TAA percentage (about 63.94 and $64.85 \%$, respectively.

Total acidic amino acid (TAAA) ranged in clover sprouts from 23.27 to 24.59 (Table 4) which was almost close to the total essential amino acid (TEAA from 23.14 to 25.44 as in Table 3). Whilst, the percentage range in total basic amino acid (TBAA) from 8.5 to 13.4 that made them the third largest amino acid group among etiolated clover sprouts samples.

Sterilized and non-sterilized seeds recorded cysteine /Total Sulfur amino acid TSAA percentage more than $50 \%$ (53.72\% and $52.68 \%$, respectively). Moreover, dry clover seeds and etiolated sprouts contain more cysteine than methionine. While opposite results was obtained using $\mathrm{NaCl}$ solution for sprouting. For examples cysteine /TSAA percentage was $62.43 \%$ in dry seeds and $56.77 \%$ in etiolated clover sprouts germinated in tap water followed by $48.1 \%$ and $36.76 \%$ in sprouts germinated in saline water (2000 ppm and 1000 ppm $\mathrm{NaCl}$, respectively). However, $\mathrm{NaCl}$ at 2000 ppm interact with non-sterilized seeds contain more cysteine than methionine. (Cysteine /TSAA percentage was $53.63 \%$ ).

Adeyeye (2004) reported that many vegetables protein contains substantially more cysteine than methionine. Similar data was obtained by Ahmed et al (2018). In contrast, animal proteins are low in cysteine (Adeyeye and Ayejuyo, 2007). However, cysteine has positive effects on mineral absorption particularly zinc (Sandstrom et al 1989 and Mendoza, 2002).

The total aromatic amino acid (TArAA) of etiolated clover sprout were ranged from $6.5 \mathrm{~g} / 100 \mathrm{~g}$ using tap water for sprouting to $6.93 \mathrm{~g} / 100 \mathrm{~g}$ using saline water (2000 ppm $\mathrm{NaCl})$ for spouting as compared with dry seeds $(7.13 \mathrm{~g} / 100 \mathrm{~g})$.

The assay methods for estimating protein quality employ casein as the reference protein. However the high quality protein used as reference should be determined at the same time and under the same conditions as the assay procedure being used FAO/WHO,(1991) therefore using standard protein (casein) in these research for estimating protein and amino acid quality is recommended even it is not identical to reference protein as reported by Tahany, Aly. (2018).

The most widely used method for measured of protein quality is protein efficiency ratio (PER) test, which is the weight gain per weight of protein eaten. The values of PER of etiolated clover sprouts sample was between 2.28 using tap water for sprouting to 2.42 using $\mathrm{NaCl} 2000$ ppm solution for sprouting compared to 2.74 in dry clover seed sample (Table 4). The higher PER value in clovers sprouts was (2.53) recorded when using non sterilized seeds sprouting using $\mathrm{NaCl} 2000$ ppm followed by $\mathrm{NaCl} 1000$ ppm (2.4).

These increments in PER value can explain by the increment in leucine and tyrosine amino acid composition (Table 3). The PER value in clover sprouts using saline water (2.53) was equal for that recorded in reference (2.5) (Oyarekua and Eleyinmi, 2004), but was higher than PER recorded in cowpea (1.21) and pigeon pea (1.82) (Salunkhe and Kadom, 1989) and green radish sprout (1.69) (Tahany, Aly. et al 2018).

Clover etiolated sprouts in both tap and saline water decreased essential amino acid index (EAAI) to $(56.45-62.21 \%)$ compared with dry seeds (68.25\%), similar to PER values. Also biological value (BV) showed similar decrement (48.77$52.86 \%)$ than dry seeds (61.63\%). However, clover sprouts using saline water increased EAAI and BV than using tap water. These results showed that consumption of etiolated clover sprouts alone as food are in adequate and in agreement with good nutritional quality (EAAI and BV less than 10 to $100 \%$ ) for food, base-protein (Oser, 1959). similar result was obtained with wheat sprout flour samples (Ibrahim, 2017) and green radish sprouts (Tahany, Aly. 2018).

Regarding nutritional index (NI), data in Table (4) showed that etiolated clover sprouts in both tap and saline water increased nutritional index to (26.98-28.31) compared with dry clover seed sample (26.32). Using non sterilized clover seed for sprouting using $\mathrm{NaCl} 2000$ ppm solution for sprouting recorded the higher nutritional index (29.86), while using $\mathrm{NaCl}$ concentration for sprouting sterilized clover seeds recorded the lower nutritional index value (26.11). 


\section{4- Effect of protein profiles}

Changing levels of protein fractions were analyzed in etiolated sprouting clover at 3 days sprout stage. Data in Table (5) should that sterilized clover seed decreased of prolamins and increased of glutelins while that of albumins and globulin fraction registered non-significant effects during sprouting. However, using saline water $(\mathrm{NaCl} 1000$ or 2000 ppm) for seed sprouting decreasing globulins and prolamins fractions while increased albumins and glutelin fractions in 3 days old etiolated sprouts as compared with using tap water for seed sprouting. The highest albumin and glutelin level was recorded using non sterilized sprouting with 2000ppm $\mathrm{NaCl}$ in traction for albumin and with $1000 \mathrm{ppm} \mathrm{NaCl}$ for glutelin, while the lower glubuling level was obtained when non -sterilized seed sprouting with $2000 \mathrm{ppm} \mathrm{NaCl}$ interaction but lower prolamin level was recorded when sterilized seed sprouting with 2000ppm $\mathrm{NaCl}$.

Finally, Albumin was the major clover sprout protein fraction extracted from $\mathrm{NaCl} 2000$ ppm sprout followed by prolamin from tap water sprouts, glutelin from $\mathrm{NaCl} 1000$ ppm sprout and globulin from tap water sprouts.

The clover sprout protein isolated and its fractions could have excellent applications for future product development by virtue of their nutritional and functional properties.

Table 5. Effect of sterilized and non-sterilized clover seed sprouting using saline water $(\mathrm{NaCl} 1000$ and $2000 \mathrm{ppm}$ ) on the protein fractionation of 3 days etiolated clover sprouts characters (mg /100mg sample)

\begin{tabular}{|c|c|c|c|c|c|}
\hline $\begin{array}{c}\text { Sterilization } \\
\text { (ste) }\end{array}$ & Parameter & Albumin & Globulin & Glutelin & Prolamin \\
\hline Sterilization & Tap water & $46.83^{\mathrm{e}}$ & $7.50^{\mathrm{a}}$ & $14.47^{\mathrm{d}}$ & $31.20^{\mathrm{a}}$ \\
NaCl, ppm & $\mathbf{1 0 0 0}$ & $52.97^{\mathrm{C}}$ & $5.73^{\mathrm{b}}$ & $18.83^{\mathrm{b}}$ & $22.47^{\mathrm{e}}$ \\
& $\mathbf{2 0 0 0}$ & $54.40^{\mathrm{b}}$ & $4.80^{\mathrm{C}}$ & $19.30^{\mathrm{b}}$ & $21.50^{\mathrm{f}}$ \\
Mean & & $51.40^{\mathrm{A}}$ & $6.01^{\mathrm{A}}$ & $17.53^{\mathrm{A}}$ & $25.05^{\mathrm{B}}$ \\
\hline Non sterilization & Tap water & $48.73^{\mathrm{d}}$ & $7.83^{\mathrm{a}}$ & $15.23^{\mathrm{C}}$ & $28.20^{\mathrm{b}}$ \\
NaCl, ppm & $\mathbf{1 0 0 0}$ & $46.20^{\mathrm{e}}$ & $6.30^{\mathrm{b}}$ & $22.20^{\mathrm{a}}$ & $25.30^{\mathrm{C}}$ \\
& $\mathbf{2 0 0 0}$ & $58.10^{\mathrm{a}}$ & $3.73^{\mathrm{e}}$ & $14.17^{\mathrm{d}}$ & $24.00^{\mathrm{d}}$ \\
Mean & & $51.01^{\mathrm{A}}$ & $5.95^{\mathrm{A}}$ & $17.20^{\mathrm{B}}$ & $25.83^{\mathrm{A}}$ \\
\hline Average & Tap water & $47.78^{\mathrm{C}}$ & $7.67^{\mathrm{A}}$ & $14.85^{\mathrm{C}}$ & $29.70^{\mathrm{A}}$ \\
NaCl, ppm & $\mathbf{1 0 0 0}$ & $49.58^{\mathrm{B}}$ & $6.02^{\mathrm{B}}$ & $20.52^{\mathrm{A}}$ & $23.88^{\mathrm{B}}$ \\
& $\mathbf{2 0 0 0}$ & $56.25^{\mathrm{A}}$ & $4.27^{\mathrm{D}}$ & $16.73^{\mathrm{B}}$ & $22.75^{\mathrm{C}}$ \\
\hline LSD 0.05 & Ste & $\mathrm{NS}$ & $\mathrm{NS}$ & 0.29 & 0.30 \\
& NaCl & 0.66 & 0.43 & 0.36 & 0.37 \\
& StexNaCl & 0.93 & 0.61 & 0.51 & 0.53 \\
\hline
\end{tabular}

Means in each column followed by the same letter are not significantly different at the $5 \%$ level $(p \leq 0.05)$. NS=non -significant.

\section{REFERENCES}

Abdallah, M.M.F., Youssef, S.M.S., Emara, M.F.Z and Ibrahim, E.M.R. 2009. Environmental and nutritional safe production of sun flower green sprouts in house. J. Environ. Sci. 19(3), 95117.

Abdallah, M.M.F. 2008. Seed sprouts, a pharaoh's heritage to improve food quality. Arab Univ. J. Agric. Sci. 16(2), 469-478.

Abd-Elghany, T.S.A. 2017. Use of organic seed sprout to improve some bakery product. M.Sc. Thesis Dept. of Env. And Bio-Agric., Fac. of Agric., Al-Azhar Univ., Cairo.115 p.
Adeyeye, E.I. 2004. The chemical composition of liquid and solid endosperm of ripe coconut. Oriental. J. of Chem., 20(3), 471-476.

Adeyeye, E.I. and Ayejuyo, 0.0. 2007. Proximate, amino acid and mineral composition of turkey-hen muscle and skin. Orient. J. Chem., 23(3), 879-886.

Ahmed, E.A.A.S., Nashwa, A.L. Abo El-Azam., El-Behairy, U.A.A. and Abdallah, M.M.F. 2018. Organic pea Sprout in door to improve pea quality. Arab Univ. J. Agric. Sci. Cairo, special Issue, 26(2), In press. 
Alsmeyer, R.H., Cunningham, A.E. and Happich, M.L. 1974. Equations predict PER from. amino acid analysis. Food Technology 7(28), 34-42.

AOAC. 2012. Official Methods of Analysis of AOAC International. $19^{\text {th }}$ ed. Dumes method. No.968.06. Chapter 4, pp. 25-26.

Cairney, E. 1997. The sprouters Handbook, Argyll Publishing Glendrauel, 2Ed edition. Argy $11 \mathrm{pA}$ 22 3E, Scotland. pp. 47-49.

Chavan, J. and Kadam, S.S. 1989. Nutritional improvement of cereals by sprouting. Critical Reviews in Food Science and Nutrition, 28(5), 401-437.

Chinma, C.E. and Igyor, M.A. 2007. Micronutrients and anti-nutritional contents of selected tropical vegetable grown in South East, Nigeria. Nig. Food. J. 25, 111-116.

Dung, D.D., Goodwin, I.R. and Nolan, J.V. 2010. Nutrient content and in Sacco digestibility of barley grain and sprouted barley. J. Anim. and Vet. Advan. 9, 2485-2492.

FAO/WHO 1991. Protein Quality Evaluation: Report of the Joint FAO/WHO Expert Consultation, FAO Food and Nutrition Paper 51, 75p.

FAO/WHO/UNU, 1985. Energy and protein requirements. WHO Technical Report Series No. 724. Geneva, 1 p.

Ghoulam, C. and Fares, K. 2001. Effect of salinity on seed germination and early seedling growth of sugar beet (Beta Vulgaris L). Seed Sci. Technol. 29, 357-364.

Guo, Y.J., XU- H.Z. and Zang, J.H. 2002. A study on the morphology of the root system of alfalfa. J. South Agric. Univ., 24, 484-486.

Han, D.L and Wang Y.R. 2005. Adaptability of medic ago sativa under water stress. Acta prataculturae Sinica, 14, 7-13.

Hasegawa, P.M., Bressan, R.A., Zhu, J.K. and Bohnert, H.J. 2000. Plant cellular and molecular responses to high salinity. Annu. Rev. Plant Physiol. Plant Mol. Biol. 51, 463-499.

Hsiao, T.C. and Xu, L.K. 2000. Sensitivity of growth of roots versus leaves to water stress: biophysical analysis and relation to water transport. Exp. Bot. 51, 1595-1616.

Ibrahim, E.M.R. 2017. Effect of sprouting using saline water on characters and chemical composition of some legumes and cereals seeds. Ph. D. Thesis, Fac. Agric. Ain shams Univ., Cairo, Egypt, pp. 40-94.

ljarorimi, S.O. and Keshinro, O.O. 2013. Determination of nutrient composition and protein quality of potential complementary foods formu- lated from the combination of fermented popcorn, African locust and Bambara groundnut seed flour. Polish J. Food. Nutr. Sci., 63(3), 155-166.

Jones, L. J.L., Thorpe, J.P. and Wallis, G.P. 1982. Genetic divergence in four species of the genus raphanus: Implication for the ancestry of the domestic radish R. Sativus. J. Linnean Society. 18(1), 35-48.

Khan, M.I.R., Mughal, A., Iqpal, N. and Khan, N.A. 2013. Potentiality of sulphur-containing compounds in salt stress tolerance. In: Ahmad P, Azooz M.M, Prasad M.N.V, editors. Ecophysiology and responses of plants under salt stress. Springer: New York, USA, pp. 443473.

Kumar, Y. and Matta, N.K. 2011. Changing protein profiles in developing and germinating barley seeds. Annals of Biological Research, 2(6), 318-329.

Labuda, J., Kacerovsky, O., Kovae, M. and Stìrba, A. 1982. Vyziva a krmenie hospodarskych zvierat. Príroda, Bratislava. 164s p.

Lang, M.M., Ingham, B.H. and Ingham, S.C. 2000. Efficacy of novel organic acid and hypochlorite treatments for eliminating Escherichia coli O157:H7 from alfalfa seeds prior to sprouting. Int. J. Food Microbiol., 58, 73-82.

Mendoza, C. 2002. Effect of genetically modified low phytic acid plants on mineral absorption, Int. J. Food Sci. Technol., 37, 759-767.

Osborne, T.B. 1909. The vegetable proteins. $\left(2^{\text {nd }}\right.$ Ed.) Longmans Green: UK, 125p.

Oser, B.L. 1959. An integrated essential amino acid index for predicting the biological value of proteins. In A.A. Albanese (ed.), Protein and amino acid nutrition. New York, USA, Academic Press. pp. 295-311.

Oyarekua, M.A. and Eleyinmi, A.F. 2004. Comparative evaluation of the nutritional quality of corn, sorghum and millet ogi prepared by a mod ified traditional technique. J. Food Agric. Environ. 2, 94-99.

Rozan, P., Kuo, Y.H. and Lambein, F. 2001. Amino acids in seeds and seedlings of the genus Lens. Phytochemistry. 58(2), 281-289.

Salunkhe, D.K. and Kadam S.S. 1989. Handbook of world food legumes, nutritional chemistry processing technology and utilization volume. Florida, USA: Boca Raton, CRC Press. 1 p.

Sandstrom, B., Almgren, A., Kivisto, B. and Cederblad, A. 1989. Effect of protein and protein source on zinc absorption in humans. J. Nutr., 119, 48-53. 
Simon-Sarkadi, L., Kocsy, G. and Sebestyen, Z. 2002. Effect of salt stress on free amino acid and polyamine content in cereals. Acta Biological szegediens. 46(3-4), 73-75.

Snedecor, G.W. and Cochran, W.G. 1980. Statistical methods $7^{\text {th }}$ ed. lowa State Univ. Press, Ames lowa, USA. 507p.

Tahany, A.A. Aly., El-Rahim, E.A., Fayed, S.A., Amal, M.A. and Abdallah, M.M.F. 2018. Influence of sprouting on chemical composition and protein quality of radish (Raphanus sativus) and clover (Trifolum alexandrinum) seeds. J. Biol. Chem. Environ. Sci., 13(1), 339-355.

Tounkara, F., Amza. T., Lagnika C., Wei Le, G. and Hui Shi, Y. 2013. Extraction, characterization, nutritional and functional properties of Roselle (Hibiscus sabdariffa Linn) seed proteins. Songklanakarin. J. Sci. Technol. 35(2), 159166.

Urbano, G., Aranda, P., Vilchez, A., Aranda, C., Cabrere, L., Pores, J.M. and Lopez-Jurado,
M. 2005. Effects of germination on the composition and nutritive value of proteins in Pisum sativum, L. Food Chemistry. 93(4), 671-679.

Wanasundara, P.K.J.P.D., Shahidi, F. and Brosnan, M.E. 1999. Changes in flax (Linum usitatissmum) seed nitrogenous compounds during germination. Food Chem., 65(3), 289295.

Wang, K.H., Lai, Y.H., Chang, J.C., Ko, T.F., Shyu, S.L. and Chiou, R.Y.Y. 2005. Germination of peanut kernels to enhance resveratrol biosynthesis and prepare sprouts as a functional vegetable. J. of Agric. and Food Chem., 53(2), 242-246.

Xue-Zhu, K., Zhou, H.M. and Qian, H.F. 2006. Proteins extracted from defatted Wheat germ: Nutritional and Structural Properties. Cereal Chem. 83(1), 69-75.

Yang, Q.C., Sun, Y., Geng, H.Z. and Guo, W.S. 2001. The proceeding of studying on alfalfa salt Tolerant Breeding and Genetic Basis. 23, 59-62. 